\title{
Determination of pKa for some Schiff bases derived from benzaldehyde and amino phenols by conductivity measurement
}

\author{
M. Gh. A. E. AL-Dabbagh \\ Department of Chemistry / College of Education \\ University of Mosul
}

Received

24 / 04 / 2012
Accepted

06 / 06 / 2012

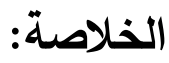

'يـعنى هذا البحث بتقدير الـ pKa لبعض قواعد شيف بواسطة الطريقة التوصيلية,

حضرت الايمينات قيد الدراسة عن طريق تفاعلات البنزالديهايد مع p,m,o امينو فينول باستخدام

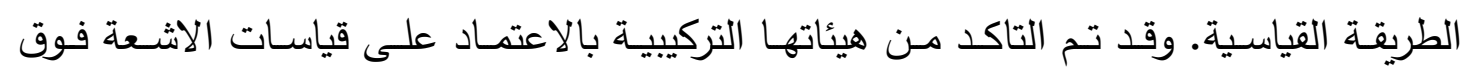
البنفجية وتحت الحمراء بالاضافة الى درجات الانصهار.

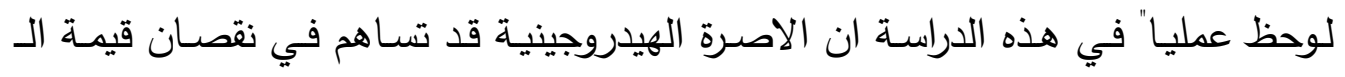

benzylidene -o-hydroxy aniline. لـ pKa مواقع p,m من جهة الامين الاولي لقواعد شيف التأثير الواضح على قيم الـ pKa المستحصلة.

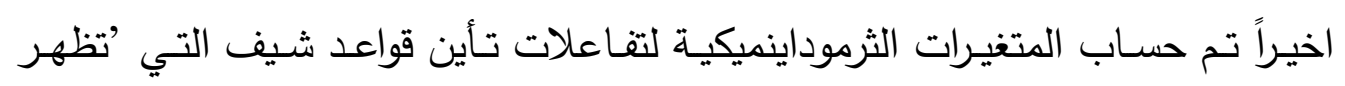

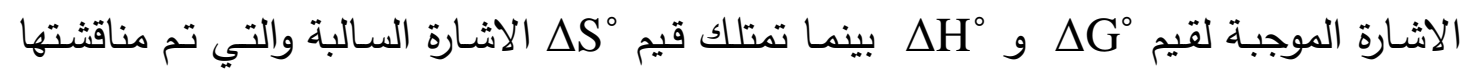
بالتفصيل.

\section{Abstract:}

The aim of this work is concerned with the determination of pka for some Schiff bases by conductivity method.These imines are prepared by reactions of benzaldehyde with o,m,p-amino phenols by standard method.

The structures of these imines are confirmed by using some physical methods, namely, U.V,I.R and melting points.

Experimentally it is observed,that intramolecular hydrogen bonding may contribute in decreasing pka value of benzylidene-ohydroxyaniline. The presence of phenolic groups at $\mathrm{m}$ and p-positions of 
primary amine side of the Schiff bases, have a noticeable influence on the collected pka values.

The thermodynamic of ionization reactions of these Schiff bases are determined. These showed a positive sign values of $\Delta \mathrm{G}^{\circ}$ and $\Delta \mathrm{H}^{\circ}$ thermodynamic parameters. Also $\Delta \mathrm{S}^{\circ}$ value has a negative sign. These positive and negative signs values are discussed in detail.

Keywords: Schiff bases,conductivity, ionization constant, pka and thermodynamic

\section{Introduction:}

Schiff bases are named ${ }^{(1)}$ with numerous methods,as ketimines when are derived from ketones and aldimines when are derived from aldehydes. These molecules have intensive importance, as theirs capability to form metal complexes. They have applications in various fields, as pharmaceutical ${ }^{(2,3,4)}$, biological $^{(5,6,7)}$ analytical $^{(8,9)}$ and industrial $^{(7,10,11)}$ applications.

Conductometric method ${ }^{(12)}$ had used extensively for determination of pka for many compounds. Ives ${ }^{(13)}$ used this method to calculate the pka of mono basic acids on the basis of determination of the equivalent conductances at the infinity dilution of their acid solutions.

Several workers had used the same method for determination of pka values for aliphatic and aromatic acids ${ }^{(14)}$, oximes and phenol derived from benzoyl acetone and dimedone ${ }^{(15)}$, formyl ${ }^{(16)}$ pipyridine and others $^{(17)}$.

The lack of pka values for benzylidene-o,m,p-substituted anilines encourage the author to deal with such important topic, using conductivity method.

\section{Experimental part:}

Chemicals:

All compounds used in this study were supplied from (Fluka), $(\mathrm{BDH})$ and (Molckula) companies. These were used directly without any extra purification process.

\section{Preparation of Schiff Bases:}

Schiff bases compounds were prepared according to a standard method $^{(18)}$, by reactions of equivalent amounts of the benzaldehyde and the primary aromatic amines. The last were in a forms of ortho, meta and para amino phenols. These reactions were carried out in(10-20)ml ethanol solvent. The final mixtures were refluxed for about $1.5 \mathrm{hr}$, cooling and recovery of the products. Purifications of products were performed by recrystallization in ethanol, followed by drying the final products. The melting points of Schiff bases were measured as shown in Table(1). 
The I.R. spectra of products under study were measured in the range between (400-4000) $\mathrm{cm}^{-1}$ and with $\mathrm{KBr}$ disc method. Also the U.V. spectra of products were measured with concentration $\left(10^{-4} \mathrm{M}\right)$ against a blank.

\section{Preparation of conductivity water:}

The usual distilled water was distillated twice to get conductivity water which has specific conductivity value of 1 to $1.2 \times 10^{-6} \mathrm{ohm}^{-1} \cdot \mathrm{cm}^{-1}$.

\section{Preparation of Sodium Hydroxide solution:}

A standard $10^{-3} \mathrm{M}$ of potassium hydrogen phthalate was prepared. This solution was titrated with sodium hydroxide using ph.ph indicator.After evaluation of concentration of unknown $\mathrm{NaOH}$, finally diluted to $10^{-3} \mathrm{M}$.

\section{Preparation of Schiff Bases Solutions:}

At the beginning a $10^{-3} \mathrm{M}$ stock solution for each acid Schiff base was prepared. This solution was diluted to prepare a set of solutions having various concentrations $(2,4,6,8) \times 10^{-4}$. The conductivites for all solutions were measured at temperatures range $(20-60){ }^{\circ} \mathrm{C}$.

\section{Preparation of the Sodium Salts of Schiff Bases:}

$10 \mathrm{ml}$ of $10^{-3} \mathrm{M}$ solution of acid Schiff bases was taken and titrated versus $10^{-3}$ of $\mathrm{NaOH}$, using ph.ph indicator. The final mixture was diluted to $50 \mathrm{ml}$ with $50 \%$ ethanol-water solvent. From the final solution other solutions mixtures were prepared of concentrations $(0.4,0.8,1.2,1.6) \times 10^{-4}$. $60)^{\circ} \mathrm{C}$.

The conductivites were measured at a range of temperatures (20-

\section{Instrumentations:}

1. The melting points were measured by using Sturart melting point apparatus, model smp30.

2. The desired temperatures under study were fixed by using a water Memmert thermostat model L200.

3. The I.R. spectra of solid Schiff bases compounds were measured by $\mathrm{KBr}$ disk method and by using a computerized Bruker Tensor- 27.

4. The U.V. spectra of ethanolic solutions of Schiff bases compounds were measured by a double beam computerized U.V.1800 Shimadzu spectrophotometer and using a matched $1 \times 1 \times 3 \mathrm{~cm}^{3}$ silica cells.

5. The conductivites were measured by using Wissenschaftlichtechnisches werkstatten model D8120-Weilheim.

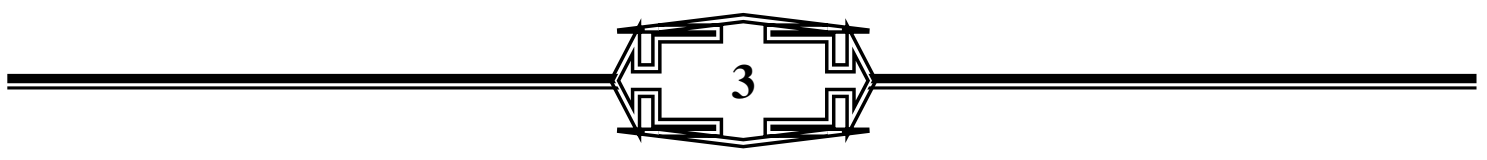


Table (1):Melting points,I.R.and U.V.spectral data for benzylidene-o,m,p-hydroxy aniline

\begin{tabular}{|c|c|c|c|c|c|c|}
\hline \multirow{2}{*}{ Symbol } & \multirow{2}{*}{ Structure } & \multirow{2}{*}{ m.p. ${ }^{\circ} \mathrm{C}$} & \multicolumn{3}{|c|}{ I.R.spectra $\mathrm{cm}^{-1}$} & \multirow{2}{*}{$\begin{array}{c}\mathrm{U} \cdot \mathrm{V} . \text { spectra } \lambda_{\mathrm{nm}}\left(\varepsilon_{\max }\right) \\
\text { in ethanol }\end{array}$} \\
\hline & & & $\mathbf{O H}$ & $\mathbf{C}=\mathbf{N}$ & Arom & \\
\hline $\mathbf{I}$ & & $89-91$ & 3328.78 & 1625.18 & 1574.10 & $435(374), 420(373), 272(1988)$ \\
\hline II & & 222decomp. & 3374.60 & 1622.95 & 1539.74 & $407(650)$ \\
\hline III & & 78-80 & 3327.25 & 1625.20 & 1574.26 & $435(840), 422(840), 280(895)$ \\
\hline
\end{tabular}




\section{Results and Discussion:}

Conductometric method had used as important sensitive, fast and accurate method for determination of pka of the mentioned acids. Previously was used in many studies to determination of pka for different acids. $^{(19)}$

For that reason the equivalent conductance for acid electrolytes must be calculated by using the following equation:

$$
\Lambda=\frac{1000 \mathrm{~K}}{\mathrm{C}}
$$

$\Lambda=$ the equivalent conductance of the electrolyte at any concentration.

$\mathrm{K}=$ the specific conductance.

$\mathrm{C}=$ the molar concentration.

There are two types of electrolytes, the strong electrolytes which give straight lines when the relationship is drawn between the equivalent conductance of electrolytes at constant temperature against square root of concentration and the weak electrolytes which give curve at the same temperature.

The equivalent conductance of sodium chloride was taken from reference $^{(20)}$ at $(20,30,40,50,60)^{\mathbf{0}} \mathrm{C}$. Similarly, also with respect of hydrochloride acid. Finally, the relationship was drawn between $\Lambda_{\mathrm{O}}$ against the mentioned temperatures as in the Figure (1). Table (2) shows $\Lambda_{\mathrm{O}}$ values with temperature range between $(20-60)^{\circ} \mathrm{C}$.

Table(2): The equivalent conductance at infinite dilution for sodium chloride and hydrochloric acid electrolyte.

\begin{tabular}{|c|c|c|}
\hline $\begin{array}{c}\text { Temperatures } \\
\left({ }^{\circ} \mathrm{C}\right)\end{array}$ & $\begin{array}{c}\Lambda_{0} \\
\mathrm{NaCl} \\
\text { ohm }^{-1} \mathrm{~cm}^{2} \text { equiv }^{-1}\end{array}$ & $\begin{array}{c}\text { Ao } \\
\text { HCl } \\
\text { ohm }^{-1} \mathbf{c m}^{2} \text { equiv }^{-1}\end{array}$ \\
\hline 20 & 117.102 & 390.204 \\
\hline 30 & 145.189 & 452.651 \\
\hline 40 & 173.276 & 515.098 \\
\hline 50 & 201.363 & $\mathbf{5 7 7 . 5 4 5}$ \\
\hline 60 & 229.45 & 639.992 \\
\hline
\end{tabular}

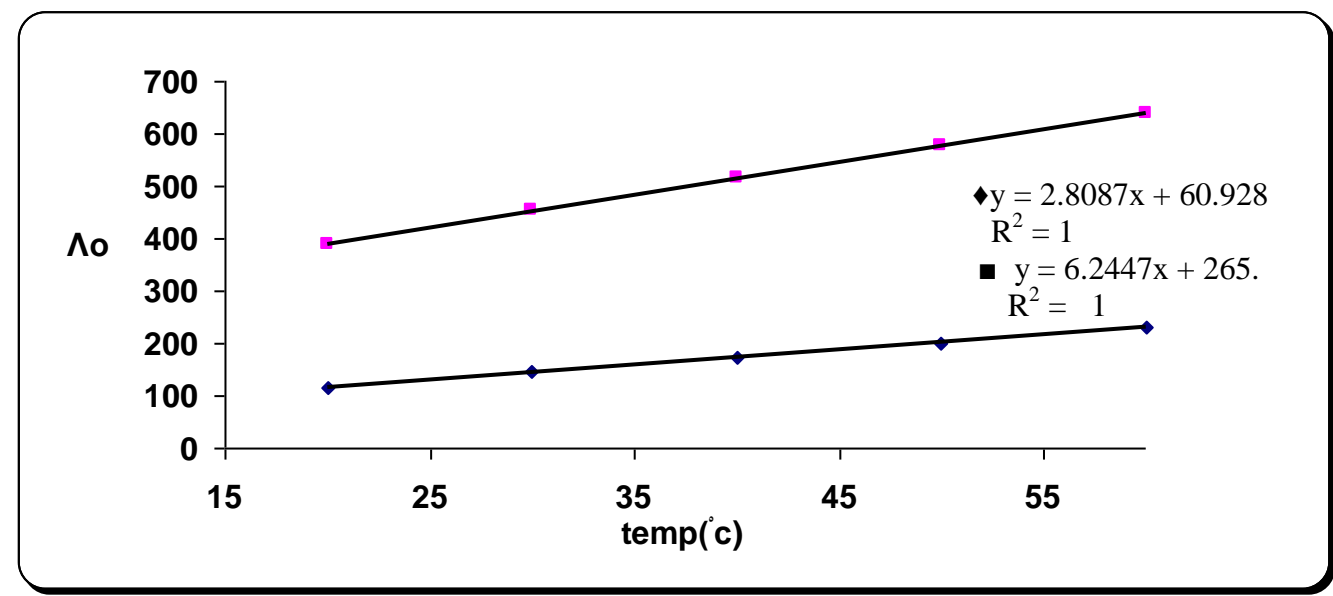

Figure(1): The relationship between $\Lambda 0$ and the centigrade temperatures of: - sodium chloride, $\square$ hydrochloric acid. 
Determination of pKa for some Schiff bases derived from benzaldehyde and ...

Different concentrations of solutions for the organic acids were prepared, the conductances for these solutions were determined and their equivalent conductances were calculated, as shown in Table (3).

Table (3): The equivalent conductances of benzylidene-o,m,p-hydroxy aniline at different temperatures.

\begin{tabular}{|c|c|c|c|c|c|c|}
\hline \multirow{2}{*}{$\begin{array}{l}\text { Compound } \\
\text { Name }\end{array}$} & \multirow{2}{*}{$\mathbf{M} \times \mathbf{x 1 0}^{-3} \sqrt{ }$} & \multicolumn{5}{|c|}{ Equivalent conductance:ohm-1 $\mathrm{cm}^{2}$ equiv $^{-1}$. } \\
\hline & & $20^{\circ} \mathrm{c}$ & $30^{\circ} \mathrm{c}$ & $40^{\circ} \mathrm{c}$ & $50^{\circ} \mathrm{c}$ & $60^{\circ} \mathrm{c}$ \\
\hline \multirow{5}{*}{$\begin{array}{l}\text { Benzylidene-o- } \\
\text { hydroxyaniline }\end{array}$} & 0.0141 & 31.50 & 42.50 & 65.00 & 80.00 & 112.50 \\
\hline & 0.0200 & 16.75 & 22.50 & 31.25 & 46.25 & 62.50 \\
\hline & 0.0245 & 8.75 & 12.83 & 16.50 & 20.00 & 26.67 \\
\hline & 0.0283 & 5.19 & 7.63 & 10.63 & 12.38 & 15.63 \\
\hline & 0.0316 & 3.35 & 4.20 & 5.25 & 6.30 & 8.30 \\
\hline \multirow{5}{*}{$\begin{array}{l}\text { Benzylidene-m- } \\
\text { hydroxyaniline }\end{array}$} & 0.0141 & 40.00 & 62.50 & 85.00 & 135.00 & 180.00 \\
\hline & 0.0200 & 15.00 & 22.25 & 22.50 & 35.00 & 53.75 \\
\hline & 0.0245 & 6.33 & 8.42 & 10.42 & 16.00 & 19.17 \\
\hline & 0.0283 & 3.38 & 4.88 & 6.63 & 8.88 & 11.50 \\
\hline & 0.0316 & 2.10 & 2.80 & 3.60 & 4.50 & 7.10 \\
\hline \multirow{5}{*}{$\begin{array}{l}\text { Benzylidene-p- } \\
\text { hydroxyaniline }\end{array}$} & 0.0141 & 30.00 & 45.00 & 77.50 & 107.50 & 145.00 \\
\hline & 0.0200 & 11.00 & 17.50 & 29.00 & 40.50 & 65.00 \\
\hline & 0.0245 & 6.25 & 9.33 & 16.50 & 23.16 & 36.00 \\
\hline & 0.0283 & 3.25 & 5.00 & 9.00 & 14.75 & 22.00 \\
\hline & 0.0316 & 1.50 & 1.80 & 3.25 & 4.50 & 6.50 \\
\hline
\end{tabular}

Similarly Table (4) show the equivalent conductance at various temperatures and concentrations of the sodium salt of organic acids under study.

Table (4): The equivalent conductances for sodium salt of benzylidene-o,m,phydroxy aniline at different temperatures

\begin{tabular}{|c|c|c|c|c|c|c|}
\hline \multirow{2}{*}{$\begin{array}{l}\text { Compound } \\
\text { Name }\end{array}$} & \multirow{2}{*}{$\mathbf{M} \mathbf{x 1 0}^{-3} \sqrt{ }$} & \multicolumn{5}{|c|}{ Equivalent conductance:ohm ${ }^{-1} \mathrm{~cm}^{2}$ equiv $^{-1}$. } \\
\hline & & $20^{\circ} \mathrm{C}$ & $30^{\circ} \mathrm{C}$ & $40^{\circ} \mathrm{C}$ & $50^{\circ} \mathrm{C}$ & $60^{\circ} \mathrm{C}$ \\
\hline \multirow{5}{*}{$\begin{array}{l}\text { Sodium salt of } \\
\text { benzylidene-o- } \\
\text { hydroxy aniline }\end{array}$} & 6.3200 & 202.5 & 210 & 250 & 325 & 400 \\
\hline & 8.9000 & 107.5 & 125 & 150 & 187.50 & 250 \\
\hline & 10.9000 & 76.67 & 91.67 & 116.67 & 150 & 191.67 \\
\hline & 12.6000 & 60.63 & 71.88 & 90.63 & 109.38 & 137.50 \\
\hline & 14.1000 & 47.50 & 60 & 77.5 & 100 & 122.50 \\
\hline \multirow{5}{*}{$\begin{array}{l}\text { Sodium salt of } \\
\text { benzylidene-m- } \\
\text { hydroxy aniline }\end{array}$} & 6.3200 & 100 & 150 & 175 & 210 & 275 \\
\hline & 8.9000 & 67.50 & 88.75 & 120 & 137.50 & 175 \\
\hline & 10.9000 & 52.50 & 61.67 & 75 & 108.33 & 150 \\
\hline & 12.6000 & 47.50 & 55.63 & 68.75 & 93.75 & 131.25 \\
\hline & 14.1000 & 41.50 & 45.50 & 65 & 90 & 110 \\
\hline \multirow{5}{*}{$\begin{array}{l}\text { Sodium salt of } \\
\text { benzylidene-p- } \\
\text { hydroxy aniline }\end{array}$} & 6.3200 & 82.50 & 202.50 & 412.50 & 600 & 900 \\
\hline & 8.9000 & 68.75 & 111.25 & 218.75 & 400 & 500 \\
\hline & 10.9000 & 48.33 & 75.83 & 125 & 166.67 & 208.33 \\
\hline & 12.6000 & 40.63 & 60 & 81.25 & 106.25 & 162.50 \\
\hline & 14.1000 & 34.50 & 50 & 60 & 97.50 & 135 \\
\hline
\end{tabular}


The relationship between $\Lambda$ against square root of the molar concentrations of electrolytic solutions of the sodium salt of the organic acids at five temperatures gave a straight lines which appears in Figure(2), the intercept of equation represents the equivalent conductance at the infinity dilution of acid salt and a different temperatures.

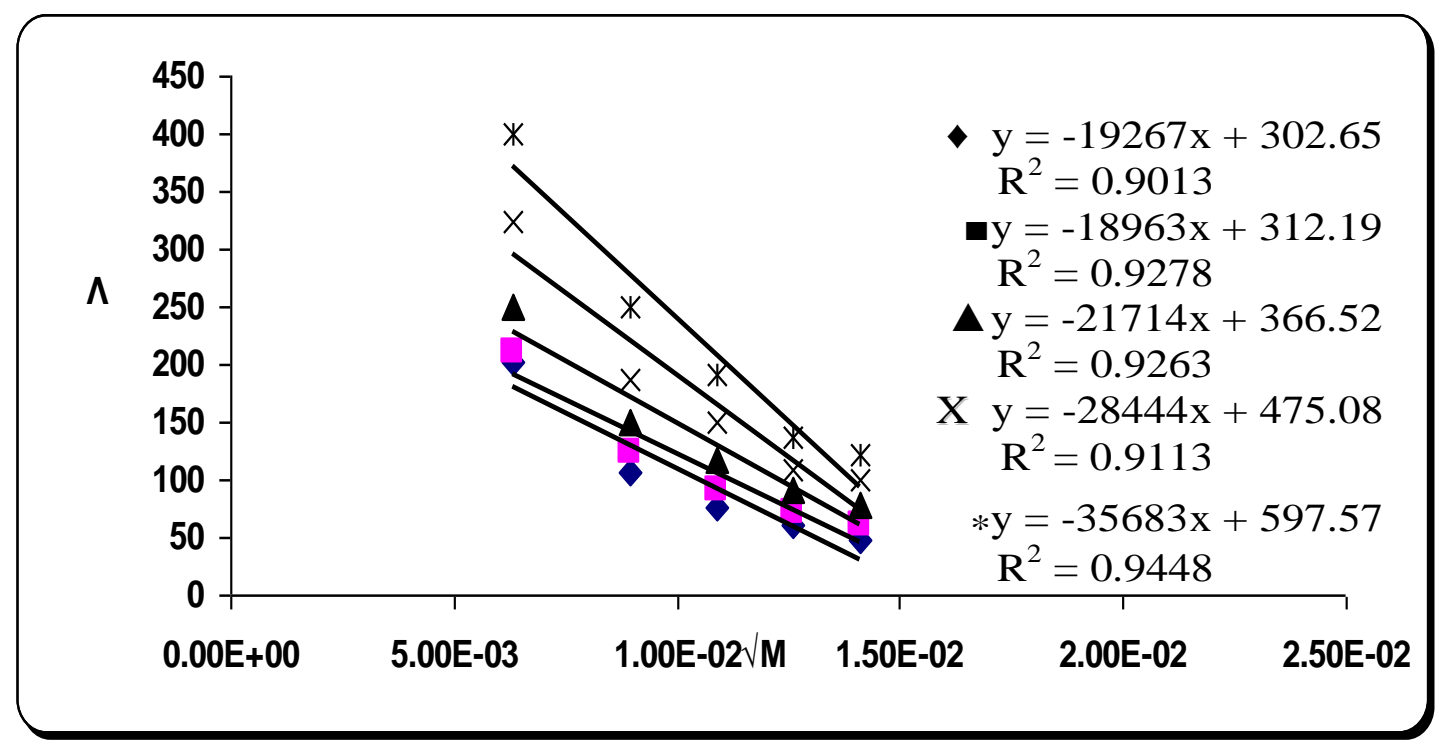

Figure (2): The relationship between $\Lambda$ versus $\sqrt{M}$ for sodium salt of BenzylideneO-hydroxyaniline at different temperatures

$(\bullet 20, \mathbf{1 3 0}, \triangle 40, \times 50, * 60){ }^{\circ} \mathrm{C}$

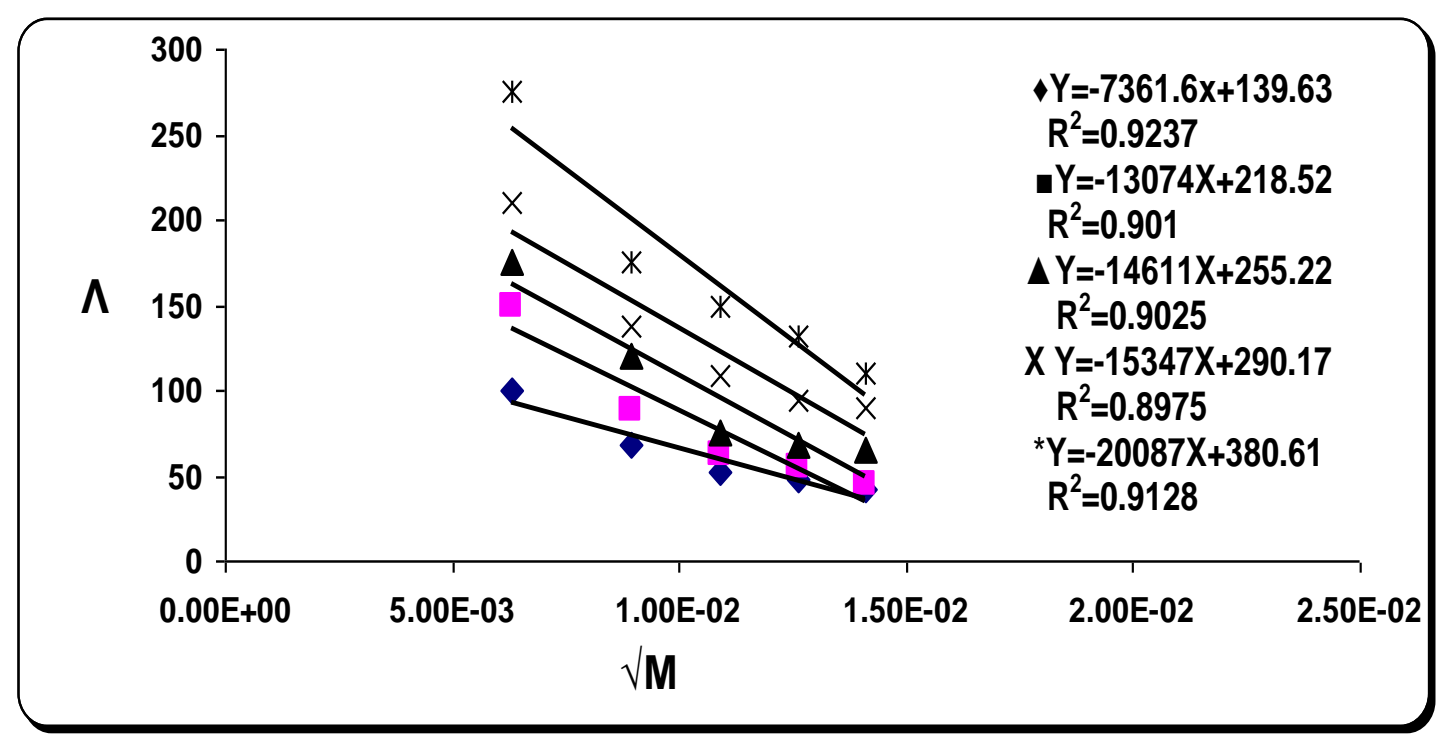

Figure (3): The relationship between $\Lambda$ versus $\sqrt{ } M$ for sodium salt of Benzylidenem-hydroxyaniline at different temperatures.

$(\diamond 20, \mathbf{3 0}, \triangle 40, \mathrm{X} 50, * 60){ }^{\mathrm{O}} \mathrm{C}$ 
Determination of pKa for some Schiff bases derived from benzaldehyde and ...

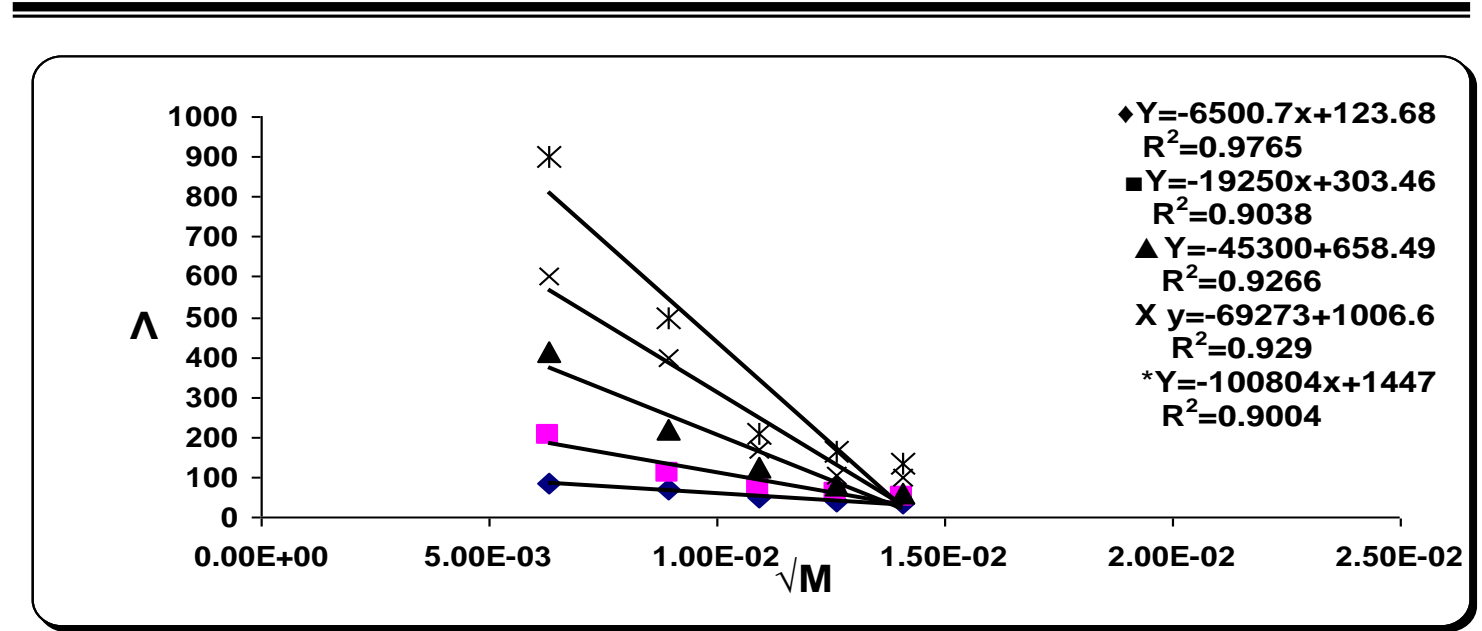

Figure (4): The relationship between $\Lambda$ versus $\sqrt{M}$ for sodium salt of Benzylidene$P$-hydroxyaniline at different temperatures.

$(\diamond 20, \mathbf{3 0}, \triangle 40, \times 50, * 60){ }^{\mathrm{O}} \mathrm{C}$

On the other hand,the relationships between equivalent conductances of organic acids under study,versus $\sqrt{\mathrm{M}}$, show a curved shapes. These finding insist ${ }^{(22)}$ (Figures 5,6 and 7) that $\Lambda_{0}$ for each acid cannot determined by this method.

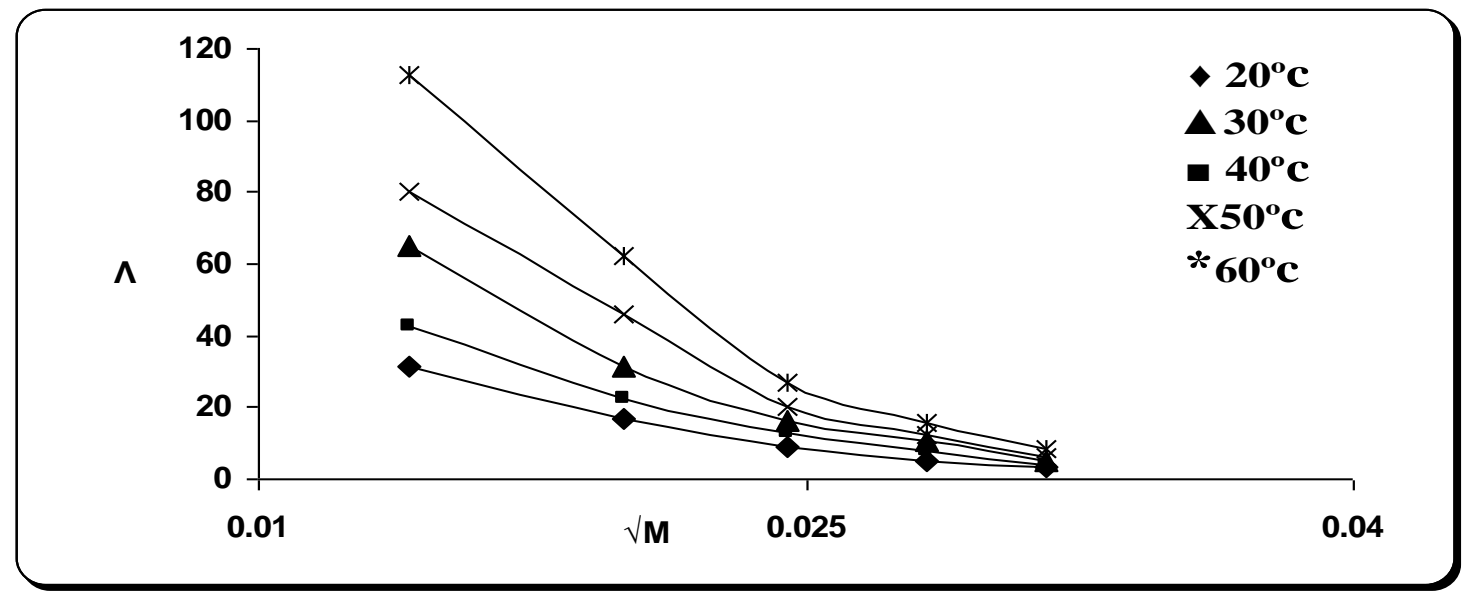

Figure (5): The relationship between $\Lambda$ versus $\sqrt{M}$ for Benzylidene-ohydroxyaniline at different temperatures

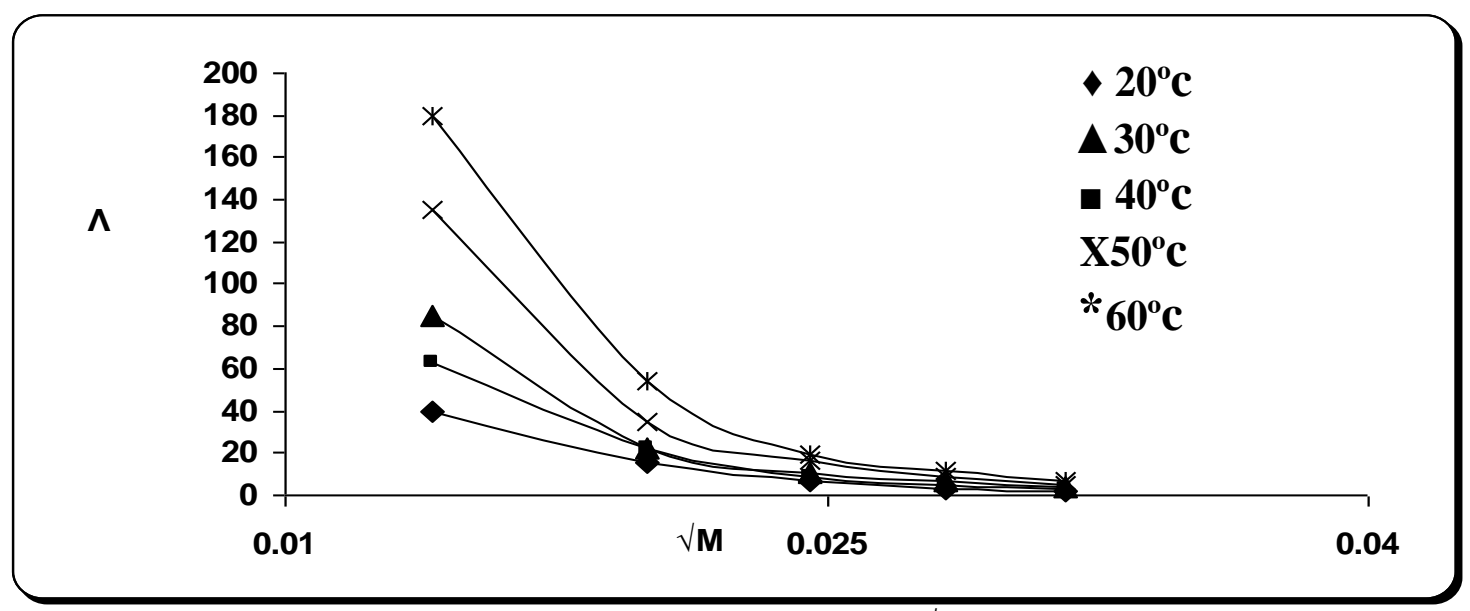

Figure (6): The relationship between $\Lambda$ versus $\sqrt{ } M$ for Benzylidene-mhydroxyaniline at different temperatures

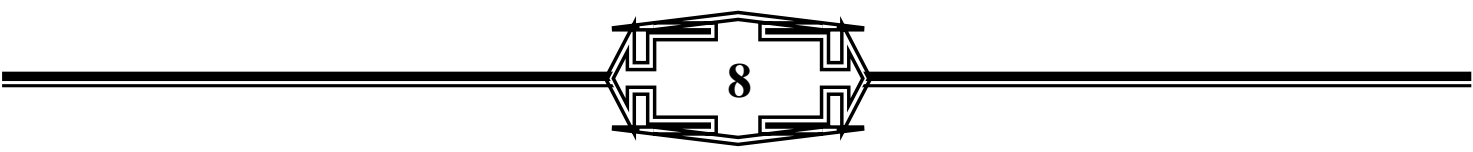




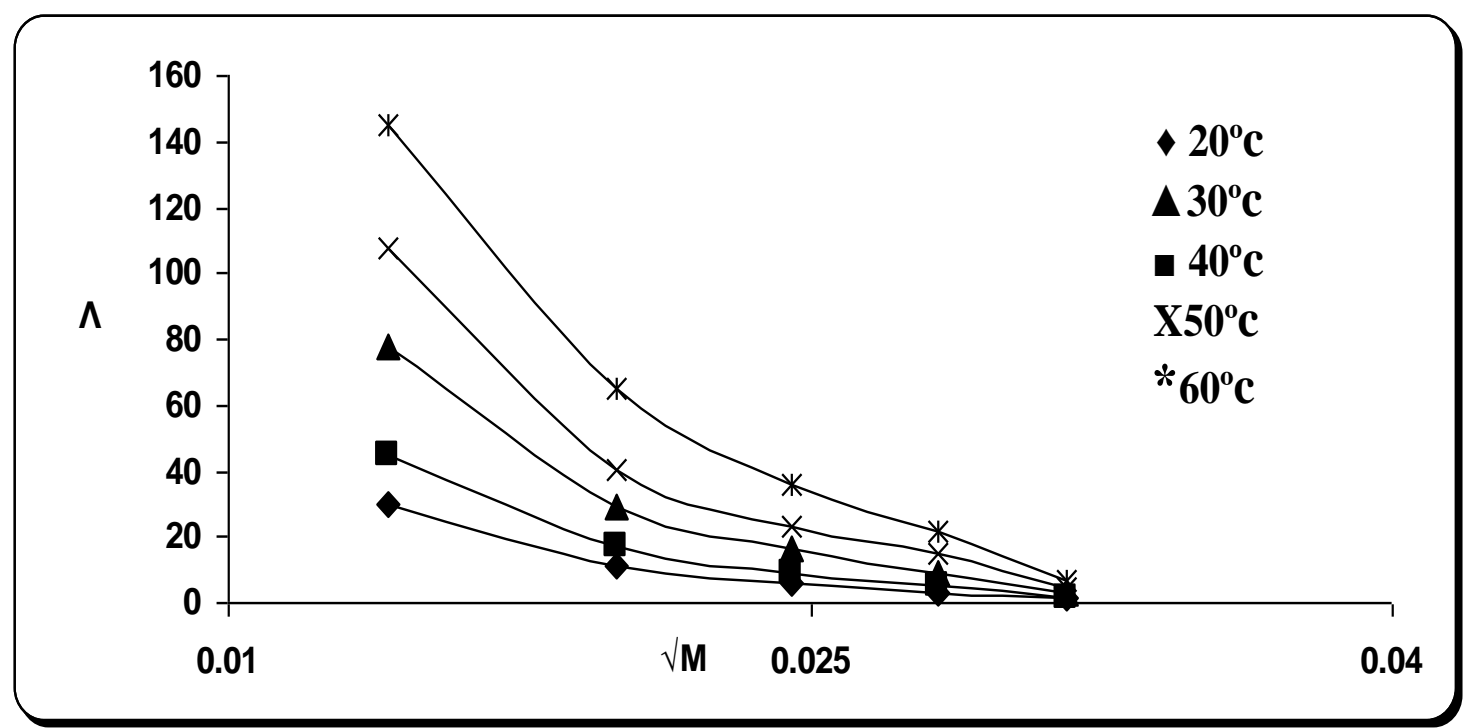

Figure (7): The relationship between $\Lambda$ versus $\sqrt{ } M$ for Benzylidene-phydroxyaniline at different temperatures

Another alternative method men,22,23) $^{(21, c o r d i n g}$ to Kohlrausch assumption was used as in the following equation as:

$\Lambda_{0}+\Lambda_{0}=\Lambda_{0}+\Lambda_{0}$

NaA HCl HA NaCl

$\Lambda_{0}=\Lambda_{0}+\Lambda_{0}-\Lambda_{0}$

$\mathrm{HA} \mathrm{NaA} \mathrm{HCl} \mathbf{N a C l}$

This facilitates the evaluation of $\Lambda_{O} \mathrm{HA}$ at any desired temperature.

Tables $(5,6,7)$ showed the equivalent conductances $\Lambda, \Lambda_{0}$, Kav and $\mathrm{pKa}$ values of acids under study.

Table(5): The equivalent conductance and pka of

Benzylidene-o-hydroxyaniline at different temperatures.

\begin{tabular}{|c|c|c|c|c|c|c|c|}
\hline $\mathbf{t}\left({ }^{\circ} \mathbf{c}\right)$ & $\begin{array}{c}\text { Conc } \times 10^{-4} \\
\text { (M) }\end{array}$ & $\begin{array}{c}\Lambda \\
\text { ohm }^{-1} \mathbf{c m}^{2} \\
\text { equiv }^{-1} \text {. }\end{array}$ & $\begin{array}{c}\Lambda_{0} \\
\text { ohm }^{-1} \\
\text { cm }^{2} \\
\text { equiv }^{-1}\end{array}$ & $\alpha \times 10^{-3}$ & $\begin{array}{c}\text { KaX10 }^{-7} \\
\text { Mole.L }{ }^{-1}\end{array}$ & $\begin{array}{c}\text { KavX10 }^{-7} \\
\text { Mole.L }^{-1}\end{array}$ & $\mathbf{p} K_{\mathrm{av}}$ \\
\hline \multirow{5}{*}{20} & 2 & 31.50 & \multirow{5}{*}{575.8} & 54.7065 & 6.3319 & \multirow{5}{*}{2.4443} & \multirow{5}{*}{6.6118} \\
\hline & 4 & 16.75 & & 29.0899 & 3.4863 & & \\
\hline & 6 & 8.75 & & 15.1962 & 1.4069 & & \\
\hline & 8 & 5.19 & & 9.0135 & 0.6559 & & \\
\hline & 10 & 3.35 & & 5.8179 & 0.3405 & & \\
\hline \multirow{5}{*}{30} & 2 & 42.50 & \multirow{5}{*}{585.3} & 72.6123 & 11.3706 & \multirow{5}{*}{4.4724} & \multirow{5}{*}{6.3495} \\
\hline & 4 & 22.50 & & 38.4418 & 6.1471 & & \\
\hline & 6 & 12.83 & & 21.9204 & 2.9476 & & \\
\hline & 8 & 7.63 & & 13.0360 & 1.3775 & & \\
\hline & 10 & 4.20 & & 7.1758 & 0.5187 & & \\
\hline \multirow{2}{*}{40} & 2 & 65.00 & \multirow{2}{*}{639.6} & 101.6260 & 22.9916 & \multirow{2}{*}{8.0112} & \multirow{2}{*}{6.0963} \\
\hline & 4 & 31.25 & & 48.8587 & 10.0396 & & \\
\hline
\end{tabular}


Determination of pKa for some Schiff bases derived from benzaldehyde and ...

\begin{tabular}{|c|c|c|c|c|c|c|c|}
\hline & 6 & 16.50 & & 25.7974 & 4.0988 & & \\
\hline & 8 & 10.63 & & 16.6198 & 2.2470 & & \\
\hline & 10 & 5.25 & & 8.2083 & 0.6793 & & \\
\hline \multirow{5}{*}{50} & 2 & 80.00 & \multirow{5}{*}{748.2} & 106.9233 & 25.6020 & \multirow{5}{*}{9.8479} & \multirow{5}{*}{6.0067} \\
\hline & 4 & 46.25 & & 61.8150 & 16.2912 & & \\
\hline & 6 & 20.00 & & 26.7308 & 4.4048 & & \\
\hline & 8 & 12.38 & & 16.5464 & 2.2270 & & \\
\hline & 10 & 6.30 & & 8.4202 & 0.7150 & & \\
\hline \multirow{5}{*}{60} & 2 & 112.50 & \multirow{5}{*}{870.7} & 129.2064 & 38.3424 & \multirow{5}{*}{13.9793} & \multirow{5}{*}{5.8545} \\
\hline & 4 & 62.50 & & 71.7813 & 22.2045 & & \\
\hline & 6 & 26.67 & & 30.6305 & 5.8071 & & \\
\hline & 8 & 15.63 & & 17.9511 & 2.6252 & & \\
\hline & 10 & 8.30 & & 9.5326 & 0.9174 & & \\
\hline
\end{tabular}

Table(6): The equivalent conductance and pka of

Benzylidene-m-hydroxyaniline at different temperatures.

\begin{tabular}{|c|c|c|c|c|c|c|c|}
\hline $\mathbf{t}\left({ }^{\circ} \mathbf{c}\right)$ & $\begin{array}{c}\text { Conc } \times 10^{-4} \\
\text { (M) }\end{array}$ & $\begin{array}{c}\Lambda \\
\text { ohm }^{-1} \mathbf{c m}^{2} \\
\text { equiv }^{-1} \text {. }\end{array}$ & $\begin{array}{c}\Lambda_{0} \\
\text { ohm }^{-1} \mathbf{c m}^{2} \\
\text { equiv }^{-1} \text {. }\end{array}$ & $\alpha \times 10^{-3}$ & $\begin{array}{c}\text { KaX10 }^{-7} \\
\text { Mole. } L^{-1}\end{array}$ & $\begin{array}{c}\text { KavX10 }^{-7} \\
\text { Mole.L }^{-1}\end{array}$ & $\mathbf{p} \mathbf{K}_{\mathrm{av}}$ \\
\hline \multirow{5}{*}{20} & 2 & 40.00 & \multirow{5}{*}{412.7} & 96.9227 & 20.8039 & \multirow{5}{*}{5.7044} & \multirow{5}{*}{6.2438} \\
\hline & 4 & 15.00 & & 36.3460 & 5.4832 & & \\
\hline & 6 & 6.33 & & 15.3380 & 1.4335 & & \\
\hline & 8 & 3.38 & & 8.1899 & 5.4103 & & \\
\hline & 10 & 2.10 & & 5.0884 & 2.6025 & & \\
\hline \multirow{5}{*}{30} & 2 & 62.50 & \multirow{5}{*}{491.6} & 127.1359 & 37.0341 & \multirow{5}{*}{9.7060} & \multirow{5}{*}{6.0130} \\
\hline & 4 & 22.25 & & 45.2604 & 8.5828 & & \\
\hline & 6 & 8.42 & & 17.1277 & 1.7908 & & \\
\hline & 8 & 4.88 & & 9.9268 & 0.7962 & & \\
\hline & 10 & 2.80 & & 5.6957 & 0.32627 & & \\
\hline \multirow{5}{*}{40} & 2 & 85.00 & \multirow{5}{*}{528.3} & 160.8934 & 61.7011 & \multirow{5}{*}{14.6807} & \multirow{5}{*}{5.8333} \\
\hline & 4 & 22.50 & & 42.5894 & 7.5783 & & \\
\hline & 6 & 10.42 & & 19.7236 & 2.3810 & & \\
\hline & 8 & 6.63 & & 12.5497 & 1.2759 & & \\
\hline & 10 & 3.60 & & 6.8143 & 0.4675 & & \\
\hline \multirow{5}{*}{50} & 2 & 135.00 & \multirow{5}{*}{563.3} & 239.6592 & 151.0891 & \multirow{5}{*}{35.0399} & \multirow{5}{*}{5.4554} \\
\hline & 4 & 35.00 & & 62.1339 & 16.4650 & & \\
\hline & 6 & 16.00 & & 28.4040 & 4.9822 & & \\
\hline & 8 & 8.88 & & 15.7642 & 2.0199 & & \\
\hline & 10 & 4.50 & & 7.9886 & 0.6433 & & \\
\hline \multirow{5}{*}{60} & 2 & 180.00 & \multirow{5}{*}{653.7} & 275.3557 & 209.2762 & \multirow{5}{*}{49.5539} & \multirow{5}{*}{5.3049} \\
\hline & 4 & 53.75 & & 82.2243 & 2.9465 & & \\
\hline & 6 & 19.17 & & 29.3254 & 5.3156 & & \\
\hline & 8 & 11.5 & & 17.5922 & 2.5202 & & \\
\hline & 10 & 7.1 & & 10.8613 & 1.1927 & & \\
\hline
\end{tabular}


Table(7): The equivalent conductance and pka of

Benzylidene-p-hydroxyaniline at different temperatures.

\begin{tabular}{|c|c|c|c|c|c|c|c|}
\hline$t\left({ }^{\circ} \mathbf{c}\right)$ & $\begin{array}{c}\text { Conc } \times 10^{-4} \\
\text { (M) }\end{array}$ & $\begin{array}{c}\Lambda \\
\text { ohm }^{-1} \mathbf{c m}^{2} \\
\text { equiv }^{-1} \text {. }\end{array}$ & $\begin{array}{c}\Lambda_{0} \\
\text { ohm }^{-1} \mathbf{c m}^{2} \\
\text { equiv }^{-1} \text {. }\end{array}$ & $\alpha \times 10^{-3}$ & $\begin{array}{c}\text { KaX10 }^{-7} \\
\text { Mole.L }\end{array}$ & $\begin{array}{c}\text { KavX10 }^{-7} \\
\text { Mole.L }^{-1}\end{array}$ & $\mathbf{p K}_{\mathrm{av}}$ \\
\hline \multirow{5}{*}{20} & 2 & 30 & \multirow{5}{*}{397.1} & 75.5477 & 12.3478 & \multirow{5}{*}{3.5395} & \multirow{5}{*}{6.4511} \\
\hline & 4 & 11 & & 27.7008 & 3.1568 & & \\
\hline & 6 & 6.25 & & 15.7391 & 1.5100 & & \\
\hline & 8 & 3.25 & & 8.1843 & 0.5403 & & \\
\hline & 10 & 1.5 & & 3.7774 & 0.1432 & & \\
\hline \multirow{5}{*}{30} & 2 & 45 & \multirow{5}{*}{576.6} & 78.0437 & 13.2128 & \multirow{5}{*}{3.8631} & \multirow{5}{*}{6.4131} \\
\hline & 4 & $\mathbf{1 7 . 5}$ & & 30.3503 & 3.8001 & & \\
\hline & 6 & 9.33 & & 16.1811 & 1.5968 & & \\
\hline & 8 & 5.00 & & 8.6715 & 0.6068 & & \\
\hline & 10 & 1.8 & & 3.1217 & 0.0775 & & \\
\hline \multirow{5}{*}{40} & 2 & 77.5 & \multirow{5}{*}{931.6} & 83.1902 & 15.0973 & \multirow{5}{*}{4.3779} & \multirow{5}{*}{6.3587} \\
\hline & 4 & 29.0 & & 31.1292 & 4.0005 & & \\
\hline & 6 & 16.50 & & 17.7115 & 1.9161 & & \\
\hline & 8 & 9.00 & & 9.6608 & 0.7539 & & \\
\hline & 10 & 3.25 & & 3.4886 & 0.1221 & & \\
\hline \multirow{5}{*}{50} & 2 & 107.5 & \multirow{5}{*}{1279.7} & 84.0041 & 15.4078 & \multirow{5}{*}{4.5494} & \multirow{5}{*}{6.3420} \\
\hline & 4 & 40.5 & & 31.6480 & 4.1371 & & \\
\hline & 6 & 23.16 & & 18.0979 & 2.0014 & & \\
\hline & 8 & 14.75 & & 11.5261 & 1.0752 & & \\
\hline & 10 & 4.5 & & 3.5164 & 0.1241 & & \\
\hline \multirow{5}{*}{60} & 2 & 145 & \multirow{5}{*}{1720} & 84.3023 & 15.5223 & \multirow{5}{*}{5.1225} & \multirow{5}{*}{6.2905} \\
\hline & 4 & 65 & & 37.7907 & 5.9370 & & \\
\hline & 6 & 36 & & 20.9302 & 2.6845 & & \\
\hline & 8 & 22 & & 12.7907 & 1.3258 & & \\
\hline & 10 & 6.5 & & 3.7791 & 0.1434 & & \\
\hline
\end{tabular}

From tables $(5,6,7)$, the following conclusions were deduced:

1- The acidity of any acid was increased by increasing temperature. This means that more protons were liberated from any acid by elevation of temperature between $(20-60){ }^{0} \mathrm{C}$. The last is accompanied by decreasing pka value of acid.

2- At any constant temperature the aciditiy was increased in the following order $m>0>p$, with exception of benzylidene-o-hydroxy aniline at temperature $293 \mathrm{~K}$. This may be due to the stronger hydrogen bonding exist at such temperature.

This order can be accepted if we considered the two opposing effect of phenolic group, namely the inductive and resonance effects. It seems possible that resonance effect of $(\mathrm{o}-\mathrm{OH})$ group is more effective as compared with inductive effect,beside the hydrogen bonding.Hence these three last effects might be responsible on decreasing the acidity of ophenolic acid as compared with m-phenolic acid at any temperature. 
The lower acidity of benzylidene-p-hydroxy aniline at any temperature can be accepted due to the stronger donation property of phenolic group at p-position supported by the $\sigma p$ value of $(-0.37)$ of Hammet equation.

These interesting results encourage the worker to deal with thermodynamic of ionization reactions of acids, namely, $\Delta \mathrm{G}^{0}, \Delta \mathrm{H}^{0}$ and $\Delta \mathrm{S}^{0}$.

$\Delta \mathrm{H}^{\mathrm{o}}$ values are calculated from integrated Vant Hoff equation at all temperatures by using the following equation:

InKa $=$ constant $-\Delta \mathrm{H}^{\mathrm{o}} / \mathrm{RT}^{(24)}$

$\mathrm{Ka}=$ ionization constant.

$\mathrm{R}=$ the gas constant $=8.314 \mathrm{~J} . \mathrm{K}^{-1} \cdot \mathrm{mole}^{-1}$.

$\mathrm{T}=$ absolute temperature.

According to this equation a linear plot was obtained on drawing Ink $_{\mathrm{a}}$ versusT $^{-1}$ with a slope equal $(-\Delta \mathrm{H} / \mathrm{R})$ as shown in Figure (8).

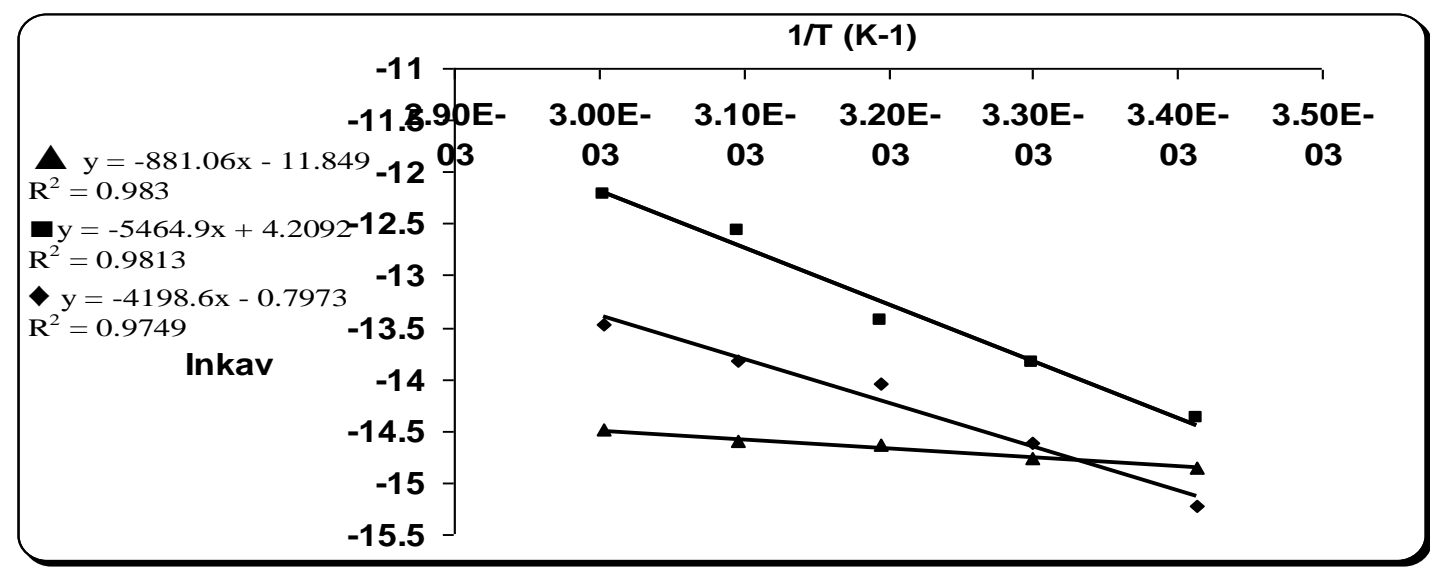

Figure(8): The relationship between $\operatorname{InK}_{\text {av }}$ against 1/T of benzylidene- $\bullet \mathbf{0 ,} \sim \mathrm{m}, \Delta \mathrm{p}$-hydroxy aniline compounds.

The positive values of heats (enthalpies) $\left(\Delta \mathrm{H}^{\circ}\right)$ indicated the ionization reactions were endothermic. These results were accepted theortically.

The change in free energy $\Delta \mathrm{G}^{0}$ for ionization reactions of acids were calculated by equation ${ }^{(25,26)}$ :

$\Delta \mathrm{G}^{0}=-\mathrm{RTInK}$.

The positive values of $\Delta \mathrm{G}^{0}$ listed as in Table (8) indicate that ionization reactions are nonspontaneous. These expected results could be accepted because all organic acids under study have covalent bonding. Thus it was difficult to ionize phenol group as compared with the ionic bonding.

$\Delta S^{0}$ values were calculated by Gibbs equation:

$\Delta \mathrm{G}^{0}=\Delta \mathrm{H}^{0}-\mathrm{T} \Delta \mathrm{S}^{0}$ 
Table (8): The thermodynamic parameters and pka of benzylidene-o,m,p-hydroxyaniline at different temperatures.

\begin{tabular}{|c|c|c|c|c|c|c|c|c|c|}
\hline $\begin{array}{l}\text { Compound } \\
\text { Name }\end{array}$ & $\underset{\mathrm{K}^{\circ}}{\text { Temp }}$ & $\begin{array}{c}\text { KavX10 }^{-7} \\
\text { Mole.L }{ }^{-1}\end{array}$ & Ink $_{\mathbf{a v}}$ & $\underset{\text { J.mol }}{\Delta \mathbf{G}^{-1}}$ & $\begin{array}{c}\Delta \mathbf{H}^{\circ} \\
\text { J.mol }^{-1}\end{array}$ & $\begin{array}{c}\Delta \mathbf{S}^{\circ} \\
\mathbf{J} \cdot \mathrm{mol}^{-1} \cdot \mathbf{K}^{-1}\end{array}$ & $\begin{array}{c}\overline{\Delta \mathbf{G}^{\circ}} \\
\text { J.mol } \\
-1\end{array}$ & $\begin{array}{c}\overline{\Delta \mathbf{H}^{\circ}} \\
\text { J.mol }\end{array}$ & $\begin{array}{c}\overline{\Delta S^{\circ}} \\
\mathbf{J} . \mathrm{mol}^{-1} \cdot \mathbf{K}^{-1}\end{array}$ \\
\hline \multirow{5}{*}{$\begin{array}{l}\text { Benzylidene- } \\
\text { o-hydroxy } \\
\text { aniline }\end{array}$} & 293 & 2.4443 & -15.2243 & 37086.43 & 35144.20 & 6.6288 & \multirow{5}{*}{36981.786} & \multirow{5}{*}{37476.29} & \multirow{5}{*}{ 6.62876- } \\
\hline & 303 & 4.4724 & -14.6202 & 36830.36 & 34821.85 & $6.6287-$ & & & \\
\hline & 313 & 8.0112 & -14.0373 & 36529.01 & 38603.81 & 6.6288 & & & \\
\hline & 323 & 9.8479 & -13.8308 & 37141.53 & 39282.62 & 6.6288 & & & \\
\hline & 333 & 13.9793 & $13.4805-$ & 37321.60 & 39528.97 & 6.6287 & & & \\
\hline \multirow{5}{*}{$\begin{array}{c}\text { Benzylidene- } \\
\text { m-hydroxy } \\
\text { aniline }\end{array}$} & 293 & 5.7044 & -14.3769 & 35022.16 & 24768.54 & $34.9953-$ & \multirow{5}{*}{34480.886} & \multirow{5}{*}{23527.36} & \multirow{5}{*}{-34.9953} \\
\hline & 303 & 9.7060 & -13.8453 & 34878.28 & 24274.70 & $34.9953-$ & & & \\
\hline & 313 & 14.6807 & -13.4316 & 34952.81 & 23999.29 & -34.9953 & & & \\
\hline & 323 & 35.0399 & -12.5616 & 33733.20 & 22429.72 & $34.9953-$ & & & \\
\hline & 333 & 49.5539 & -12.2150 & 33817.98 & 22164.55 & $34.9953-$ & & & \\
\hline \multirow{5}{*}{$\begin{array}{c}\text { Benzylidene- } \\
\text { p-hydroxy } \\
\text { aniline }\end{array}$} & 293 & 3.5395 & -14.8541 & 36184.62 & 65048.81 & -98.5126 & \multirow{5}{*}{38160.364} & \multirow{5}{*}{68994.80} & \multirow{5}{*}{ 98.5126- } \\
\hline & 303 & 3.8631 & -14.7666 & 37199.16 & 67048.48 & -98.5126 & & & \\
\hline & 313 & 4.3779 & -14.6415 & 38101.31 & 68935.75 & -98.5126 & & & \\
\hline & 323 & 4.5494 & -14.6031 & 39215.49 & 71035.05 & -98.5126 & & & \\
\hline & 333 & 5.1225 & -14.4845 & 40.101 .24 & 72905.92 & 98.5126- & & & \\
\hline
\end{tabular}

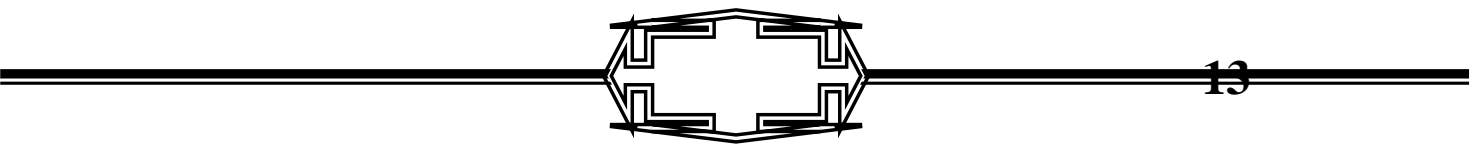


Table(8) shows that the $\Delta S^{0}$ values have negative signs. This means that, the ionization products are less random as compared with organic reactants. This was possible for bases having a hydrogen bonding stated before.

All these results were in agreement with other earlier study in the same field ${ }^{(16,17,27)}$.

\section{Conclusions:}

1. The conductivities of phenolic acids were increased with increasing of temperatures.

2. The conductivities of phenolic sodium salts of Schiff bases were increased with increasing of temperatures.

3. Generally the acidity of acid phenols was increased with increasing of temperatures.

4. At any constant temperature the acidity of acid phenols was increased in the following order $m>0>p$, with exception of benzylidene-o-hydroxy aniline at temperature $293 \mathrm{~K}$. This may be due to the stronger hydrogen bonding exist at such temperature.

\section{References:}

1) R.W.Layer., Chem. Rev., 63:489 (1963).

2) A.Kulshrestha and S.Baluja., Scholars Research Library., 2(3):221224 (2010).

3) S.Aureus, E.Coli, Styphi, C.Albicans, Rhizopussp, A.Niger, Organic Chemistry International, Article, ID, (2010), 648589, 7 pages.

4) N.Raman, J.D. Raja and A.Sakthival., J. Chem. Sci., Vol. 119: 303310 (2007).

5) K.Kiranmai, Y. Prashanthi, N. J. P. Subhashini and Shivaraj., J. Chem. Pharm. Res., 2(1):375-384 (2010).

6) Suparna Ghosh, Suman Malik, Bharti Jain and N. Ganesh, Asian., J. Exp. Sci., Vol. 23, No. 1:189-192 (2009).

7) Shalin Kumar, Durga Nath Dhar and P.N. Saxena., Journal of Scientific and Industrial Research., Vol. 68: 181-187(2009).

8) P.J. Clark, I.E.Treble and P.C.Vden., Polyhedron., 1:785 (1982).

9) R.A. Khalil, A. H. Jalil and A. Y. Abd-Alrazzak., J. Iran. Chem. Soc., Vol. 6, No. 2: 345-352 (2009).

10) A.A. Isse, A. Gennaro and E. Vianello., J. Electronal. Chem., 444: 241-245 (1998).

11) A.R. Oki and D.J. Hodgson., Inorg. Chem. Acta., 170: 65 (1990).

12) A. Albert and E.P. Serjeant., "The Determination of Ionization constant". $3^{\text {rd }}$ ed, Chapman and Hall, London, New York; 128-207 (1984). 
13) D. J. G. Ives., J. Chem. Soc., 731 (1933).

14) Samik Nag and Dipankar Datta., Ind. J. Chem., Vol. 46A: 12631265 (2007).

15) A.S.P.Azzouz and F.H. Maree., J. Edu. Sci., 21(1), 1 (2008).

16) A.S.P. Azzouz and H.J. Al-Mula., Al-Anbar J. Pure Sciences., 4(2), 59 (2010).

17) A.S.P. Azzouz and M.A. Hussien., J. Edu. Sci., 24(4), 1 (2011).

18) E.D.S. Pati, "The Chemistry of Carbon - Nitrogen Double Bond". John Wiley and Sons, New York, pp. 50-86, 162-164, 365-385 (1979).

19) A.S.P.Azzouz and S.S.Othman., J.Edu.Sci., 26:86 (1997).

20) R.C. Weast, "Hand Book of Chemistry and Physics". 55th. Published by CRC Press, pp. 132-134 (1974),

21) G.M.Barrow, "Physical Chemistry". 5th ed. McGraw -Hill Book Company, Inc., New York, pp.306-313, (1988).

22) A. Martin, "Physical Pharmacy". 4th ed. Lea and Febiger, London, p.260 (1993).

23) J.F.J. Dippy, S.R.C. Hughes and J.W. Laxton., J. Chem. Soc., 1470 (1954).

24) A.S.P. Azzouz and Kh.I. Al-Niemi., J. Edu. Sci., 16, 59 (2004).

25) J. Hine, "Physical Organic Chemistry". McGraw-Hill Book Company, Inc., London, p.33 (1962).

26) ليلى محمد نجيب، محمود شاكر سعيد، "الكيمياء الفيزيائية"، دار الكتب للطباعة والنشر،

$$
\text { جامعة الموصل (1989). }
$$

27) N.A. Al-Azzawi., Ph.D. Thesis, College of Education, University of Mosul, $\operatorname{Iraq}(1998)$. 